\title{
The Coup-TFII orphan nuclear receptor is an activator of the $\gamma$-globin gene
}

\author{
Cristina Fugazza,,$^{1^{*}}$ Gloria Barbarani, ${ }^{1^{*}}$ Sudharshan Elangovan, ${ }^{1^{* 0}}$ Maria \\ Giuseppina Marini, ${ }^{2}$ Serena Giolitto, ${ }^{1}$ Isaura Font-Monclus, ${ }^{1}$ Maria Franca \\ Marongiu, ${ }^{2}$ Laura Manunza, ${ }^{3}$ John Strouboulis, ${ }^{4}$ Claudio Cantù, ${ }^{5}$ Fabio \\ Gasparri, ${ }^{6}$ Silvia M.L. Barabino, ${ }^{1}$ Yukio Nakamura, ${ }^{7}$ Sergio Ottolenghi, ${ }^{1}$ Paolo \\ $\mathrm{Moi}^{3}$ and Antonella Ellena Ronchi ${ }^{1}$
}

Haematologica 2021

Volume 106(2):474-482

\section{Correspondence:}

ANTONELLA ELLENA RONCHI1 antonella.ronchi@unimib.it

Received: October 30, 2019.

Accepted: February 20, 2020.

Pre-published: February 27, 2020.

https://doi.org/10.3324/haematol.2019.241224

(C)2021 Ferrata Storti Foundation

Material published in Haematologica is covered by copyright. All rights are reserved to the Ferrata Storti Foundation. Use of published material is allowed under the following terms and conditions:

https://creativecommons.org/licenses/by-nc/4.0/legalcode. Copies of published material are allowed for personal or internal use. Sharing published material for non-commercial purposes is subject to the following conditions:

https://creativecommons.org/licenses/by-nc/4.0/legalcode, sect. 3. Reproducing and sharing published material for commercial purposes is not allowed without permission in writing from the publisher.
${ }^{1}$ Dipartimento di Biotecnologie e Bioscienze, Università degli Studi di Milano-Bicocca, Milano, Italy; ${ }^{2}$ Istituto di Ricerca Genetica e Biomedica del Consiglio Nazionale delle Ricerche, Cagliari, Italy; ${ }^{3}$ Dipartimento di Sanità Pubblica, Medicina Clinica e Molecolare, Università degli Studi di Cagliari, Cagliari, Italy; ${ }^{4}$ School of Cancer and Pharmaceutical Sciences, King's College London, London, UK; ${ }^{5}$ Wallenberg Center for Molecular Medicine, Department of Clinical and Experimental Medicine (IKE), Linköping University, Linköping, Sweden; ${ }^{6}$ Department of Biology, Nerviano Medical Sciences S.r.l., Nerviano, Milano, Italy and ${ }^{7}$ University of Tsukuba, Tsukuba, Ibaraki, Japan

${ }^{\star} \mathrm{CF}, \mathrm{GB}$ and $\mathrm{SE}$ contributed equally as co-first authors.

- Present address: Genomics Division, Wipro Life Sciences Laboratory, WIPRO Limited, Bengaluru, Karnataka, India

\section{ABSTRACT}

T he human fetal $\gamma$-globin gene is repressed in adulthood through complex regulatory mechanisms involving transcription factors and epigenetic modifiers. Reversing $\gamma$-globin repression, or maintaining its expression by manipulating regulatory mechanisms, has become a major clinical goal in the treatment of $\beta$-hemoglobinopathies. Here we identify the orphan nuclear receptor Coup-TFII (NR2F2/ARP1) as an embryonic/fetal stage activator of $\gamma$-globin expression. We show that Coup-TFI is expressed in early erythropoiesis of yolk sac origin, together with embryonic/fetal globins. When overexpressed in adult cells (including peripheral blood cells from human healthy donors and $\beta 039$ thalassemic patients) Coup-TFII activates the embryonic/fetal globin genes, overcoming the repression imposed by the adult erythroid environment. Conversely, the knockout of Coup-TFII increases the $\beta / \gamma+\beta$ globin ratio. Molecular analysis indicates that Coup-TFII binds in vivo to the $\beta$-locus and contributes to its three-dimensional conformation. Overall, our data identify Coup-TFII as a specific activator of the $\gamma$ globin gene.

\section{Introduction}

In mammals, the developmental regulation of erythropoiesis ensures the production of the different types of hemoglobin, from embryonic to fetal and adult, required at the different developmental stages. In humans, the switch from $\gamma$ - to $\beta$ globin expression, resulting in the change from fetal hemoglobin ( $\mathrm{HbF})$ to adult hemoglobin $(\mathrm{HbA})$ production, is the subject of intense investigation. In fact, the maintenance of expression of the fetal $\gamma$-globin gene in the adult can compensate for defects in $\beta$-globin synthesis causing $\beta$-hemoglobinopathies, the most common monogenic inherited human diseases. ${ }^{1.5}$ This evidence has focused the research on discovering $\gamma$-globin repressors in adult cells, several of which have been identified to-date, the best example being the transcriptional repressor Bcl11a-XL. ${ }^{6}$ By contrast, less is known about possible specific activators of embryonic/fetal genes in early developmental stages.

Early murine erythropoiesis is sustained by two waves of cells of yolk sac origin. The first wave generates "primitive" red blood cells (EryP), which synthesize embryonic $\varepsilon Y$ and $\beta H 1$-globins; the second wave produces erythro-myeloid progenitors (EMP), which colonize the fetal liver and differentiate into erythroid and 
myeloid cells. EMP-derived erythroid progenitors later on will generate the first "definitive" erythroid cells, which synthesize mainly adult $\beta^{\text {maj }}$ and $\beta^{\text {min }}$ globins and little $\beta \mathrm{H} 1$, prior to the emergence of the "adult" aorta-gonadmesonephros-derived hematopoietic stem cells. ${ }^{7.8}$ In mice transgenic for the human $\beta$-locus, EryP synthesize $\varepsilon$ - and $\gamma$-globin chains, whereas the EMP erythroid progeny mainly expresses $\gamma$ - and some $\beta$-globin; hematopoietic stem cell-derived definitive cells express human $\beta$ - and very little, if any, $\gamma$-globin. ${ }^{8}$

In this work, we focused on the chicken ovalbumin upstream promoter-transcription factor II (Coup-TFII or NR2F2/ARP1). Coup-TFII is an orphan nuclear receptor essential for many biological processes, as demonstrated by early embryonic death of Coup-TFI knockout mice. ${ }^{9}$ Conditional knockout models further demonstrated the requirement of COUP-TFI in different developmental programs, such as angiogenesis, organogenesis, cell fate determination and metabolic homeostasis. ${ }^{10}$ Within the erythroid lineage, Coup-TFI is expressed in the early embryo and its levels decline around embryonic day E12.5 ${ }^{11,12}$

Coup-TFII was originally identified as part of the NF-E3 complex, proposed to act as a repressor of $\varepsilon$ - and $\gamma$-globin genes on the basis of in vitro DNA binding studies. ${ }^{11,13,14}$ Subsequently, transfection experiments showed that the binding of Coup-TFII to both $\varepsilon$ - and $\gamma$-globin promoters interferes with that of NF-Y and possibly of other transcription factors and/or cofactors, ${ }^{15-17}$ resulting in either cooperation or competition. ${ }^{15}$

Here we show that Coup-TFII is co-expressed with embryonic globins in cells of yolk sac origin. Significantly, Coup-TFII overexpression induces $\gamma$-globin (and $\beta \mathrm{H} 1$ ) in different adult erythroid cellular systems. At the molecular level, overexpression or knockout of Coup-TFII in $\beta$ K562 cells ${ }^{18}$ (to our knowledge the only erythroid model system expressing Coup-TFII), alters the $\gamma / \beta$ expression ratio and the frequencies of the interactions within the $\beta$ locus in opposite directions, further demonstrating that Coup-TFII favors the expression of embryonic/fetal globins.

\section{Methods}

\section{Cell cultures}

$\beta$-K562 cells were cultured in RPMI with $10 \%$ fetal bovine serum. ${ }^{18}$ Ex vivo cultures of mouse E13.5 fetal liver were established from human $\beta$-locus transgenic mice (a gift from Prof. Frank Grosveld). After expansion of erythroblasts, cells were infected, with a multiplicity of infection of 100 , and analyzed $72 \mathrm{~h}$ after transduction. ${ }^{19}$ Human burst-forming unit erythroid cultures from peripheral blood were obtained essentially as described by Migliaccio et al. ${ }^{20}$ by using a two-phase protocol (details in the Online Supplementary Methods). Cells were monitored by flow cytometry for the expression of CD71, Ter119/CD235a, CD117 and $\triangle \mathrm{NGFR}$. Experiments on mouse and human samples were approved by the Italian Ministry of Health.

\section{Coup-TFIl overexpression}

Coup-TFII murine cDNA (a gift from Dr. Michèle Studer) was cloned into a bicistronic IRES-eGFP or IRES- $\triangle$ NGFR CSI vector (a gift from Prof. Tariq Enver) (Online Supplementary Figure S1). Exogenous expression was monitored by quantitative real-time polymerase chain reaction (RTqPCR) analysis and/or western blotting and flow cytometry (Figure 3 and Online Supplementary Figures S2, 3 and 5).

\section{Coup-TFII CRISPR/Cas9 knockout}

The 39900 single guide RNA from the GeCKOv2 library (https://www.addgene.org/crispr/libraries/geckov2) was cloned into the LentiCrisprV2 plasmid (Addgene \#52961), as described in the Online Supplementary Methods. After selection, single knockout (KO) cells were isolated to obtain single clones.

\section{Lentiviral production and transduction experiments}

HEK-293T cells were transfected with the Coup-TFII or empty vector plus the packaging plasmids psPAX2 and pMD-VSVG (www.lentiweb.com). Seventy-two hours after transfection, recombinant particles were collected, concentrated and resuspended in phosphate-buffered saline.

\section{RNA isolation and polymerase chain reaction}

The procedures used for RNA isolation and RTqPCR are detailed in the Online Supplementary Methods.

\section{Western blotting}

Protein extracts were prepared according to standard protocols ${ }^{21}$ and subjected to sodium dodecylsulfate polyacrylamide gel electrophoresis and blotting.

\section{Immunofluorescence}

Cells were fixed in $4 \%$ paraformaldehyde, washed, permeabilized and incubated overnight with the appropriate antibodies. DAPI was added 20 min before the final wash in phosphatebuffered saline. Cells were analyzed using an ArrayScan VTI highcontent screening reader (Thermo-Fisher), as described by Durlak et al. ${ }^{18}$ or by flow cytometry.

\section{Chromatin immunoprecipitation, sequencing and analysis}

$\beta$-K562 overexpressing Coup-TFII were fixed with $1 \%$ formaldehyde for $10 \mathrm{~min}$ at room temperature. Chromatin was sonicated to a size of about 500 bp. DNA immunoprecipitation was obtained by incubation with the appropriate antibodies and subsequent isolation with protein A-agarose beads. DNA was sequenced on an Illumina platform. The sequencing data were uploaded to the Galaxy web platform. ${ }^{22}$ Reads were mapped to the human genome (GRCh37/hg19) using the Burrows-Wheeler aligner $^{23}$ and peaks were detected using the HOMER tool package..$^{24}$ Peaks in different experiments were called as the same if the minimal overlap of intervals was $1 \mathrm{bp}$. To identify peaks bound in one experiment but not in another, intervals should not overlap. ${ }^{25,26}$ Motif discovery was conducted using Multiple Expression motifs for Motif Elicitation (MEME) ${ }^{27}$ with default parameters.

\section{Chromosome conformation capture}

Chromosome conformation capture (3C) experiments ${ }^{28,29}$ are described in detail in the Online Supplementary Methods.

\section{Flow cytometry}

Transduced cells were washed in phosphate-buffered saline, fixed in $4 \%$ paraformaldehyde, stained with appropriate antibodies, acquired on FACSCalibur (Becton-Dickinson) or a CytoflexS (Beckman-Coulter) and analyzed with Summit V4.3 software or CytExpert, respectively.

\section{Primers, antibodies and reagents}

The primers, antibodies and reagents used in this study are listed in Online Supplementary Tables S1 and S2. 
A

E11.5 E12.5 E13.5

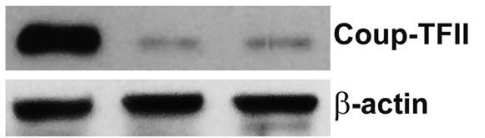

B
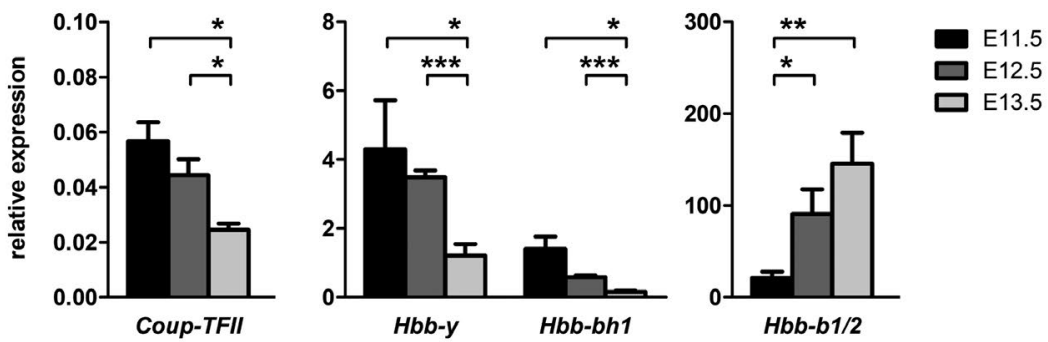

C

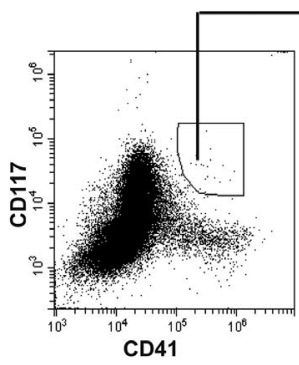

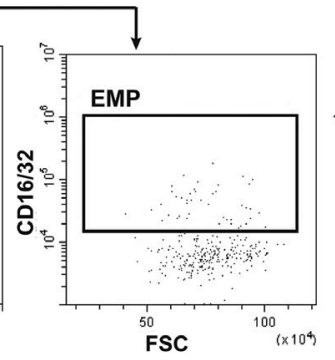

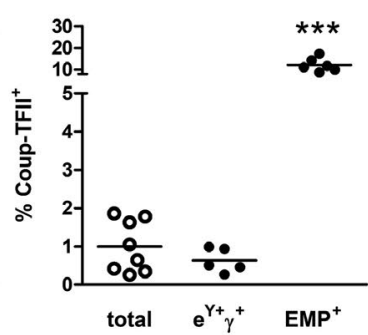

D
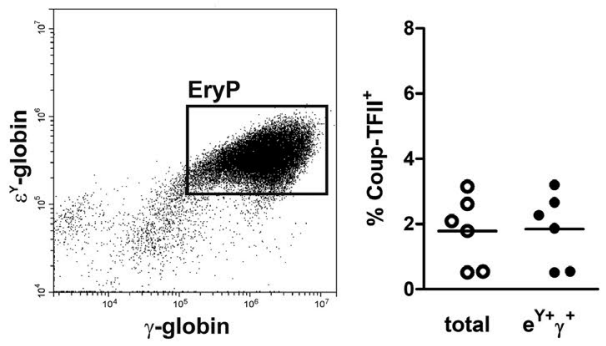

Figure 1. Expression profile of Coup-TFII during the early fetal liver globin switching developmental window. (A) Representative western blot to assay the protein lev els of Coup-TFII in nuclear extracts from E11.5, E12.5 and E13.5 mouse fetal livers. $\beta$-actin was used as a loading control. (B) Quantitative real-time polymerase reaction expression analysis relative to glyceraldehyde-3-phosphate dehydrogenase (Gapdh) $(\mathrm{n} \geq 4$, error bars: standard error of mean; $* P<0.05$; $* * P<0.01$; $* * * P<0.001$ ). (C) Flow cytometry (FC) analysis defining Coup-TFIl in erythro-myeloid progenitors (CD41 $/$ CD $\left.117^{+} / \mathrm{CD} 16 / 14^{+}\right)$and "primitive" red blood cells (EryP: ey $\left.{ }^{+} \gamma^{+}\right)$in fetal liver cells from human $\beta$-globin locus transgenic mouse embryos at E11.5. (D) FC analysis identifying Coup-TFII in EryP ( $\left(\varepsilon y^{+} \gamma^{+}\right)$in blood at E11.5. FSC: forward scatter; EMP: erythro-myeloid progenitors.

\section{Statistical analysis}

Data are expressed as the mean \pm standard error of mean of $\geq 3$ replicates and were analyzed using a paired two-tailed Student ttest (GraphPad Prism)

\section{Results}

\section{Coup-TFIl expression declines during the time of hemoglobin switching}

To gain insight into the role of Coup-TFII in the process of hemoglobin switching, we first analyzed its expression in mouse fetal liver at E11.5-E13.5, i.e., in the timeframe during which globin switching takes place. Coup-TFII is expressed at E11.5 and both its protein and RNA levels decline sharply (Figure 1A, B) at E12.5-E13.5; its expression profile follows that of the decline of embryonic mouse Hbb-bht/Hbb-y globins and the parallel accumulation of adult Hbb-b1/2 globins (Figure 1B). This trend is consistent with a potential role of Coup-TFII as an activator of embryonic globins, which is the subject of this work.

In order to investigate the role(s) of Coup-TFII in embryonic/fetal to adult hemoglobin switching in greater detail, we took advantage of a mouse model that is transgenic for the entire human $H B B$ locus ${ }^{30}$ in which the human $\gamma$ to $\beta$ switch occurs in parallel to the embryonic/adult switch of the murine genes.

In E11.5 fetal liver, erythropoiesis is sustained by EryP cells derived from the yolk sac which express murine embryonic $\varepsilon Y$ and $\beta \mathrm{H} 1$ globins and human transgenic $\varepsilon$ and $\gamma$-globins, ${ }^{8}$ and by EMP that will generate the first definitive red blood cells expressing adult $\beta$-globins. ${ }^{7,8}$
The overall percentage of Coup-TFII ${ }^{+}$cells in the E11.5 fetal liver is about $2 \%$ (Figure 1C). Only approximately $0.63 \%$ of EryP cells (i.e., $\mathrm{ey}^{+} \gamma^{+}$) cells express Coup-TFII, whereas approximately $12.2 \%$ of the rare $(0.06 \%)$ EMP population, identified as $\mathrm{CD} 41^{+} / \mathrm{CD} 117^{+} / \mathrm{CD} 16 / 32^{+}$cells, ${ }^{7}$ express it (Figure 1C). In blood, about $97.7 \%$ of cells are $\varepsilon^{+} \gamma^{+}$, but only a small percentage of them $(1.79 \%)$ also express Coup-TFII (Figure 1D). This suggests asynchrony between Coup-TFII expression and hemoglobin synthesis, Coup-TFII being predominantly expressed at early stages and then declining during maturation and hemoglobinization. Accordingly, the few $\varepsilon y^{+} \gamma^{+}$Coup-TFII cells found in the fetal liver could possibly represent EryP progenitors undergoing maturation.

\section{Coup-TFIl overexpression activates embryonic/fetal glo- bin genes in adult cells}

To test whether Coup-TFI activates embryonic/fetal globin genes, we first reintroduced Coup-TFII into fetal liver cells isolated from mouse embryos transgenic for the human $\beta$-locus at E13.5, when Coup-TFI expression is already greatly reduced (Figure 1). We transduced the expanded proerythroblasts from E13.5 fetal liver with viral particles carrying either the Coup-TFII- $\Delta$ NGFR overexpressing vector, or the empty vector EV- $\Delta$ NGFR control (Online Supplementary Figure S1). As shown in Figure 2A, Coup-TFII significantly increased human $\gamma$-globin and mouse $\beta \mathrm{H} 1$ expression, without affecting the expression of mouse (Hbb-b1/2) and human (HBB) adult globins. This resulted in a significantly increased $\gamma /(\gamma+\beta)$ globin ratio. No major changes were observed in $\alpha$-like mouse globin genes (Online Supplementary Figure S2). 

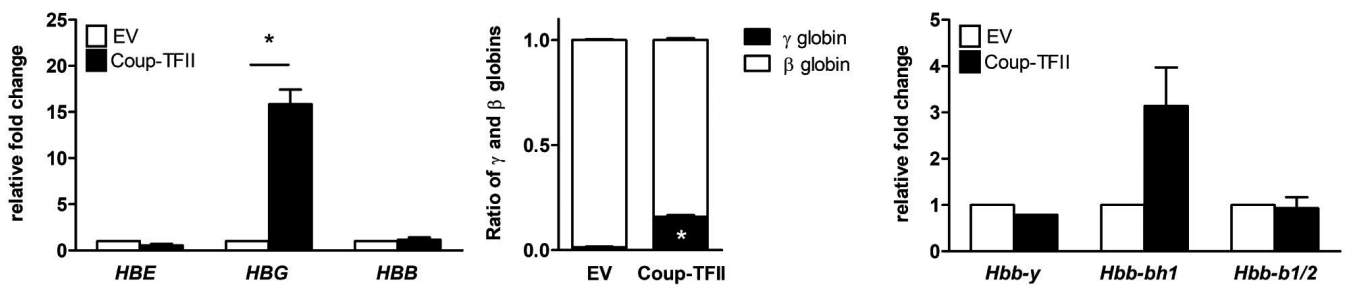

B

human healthy donor

human thalassemic $\beta^{039}$ patient
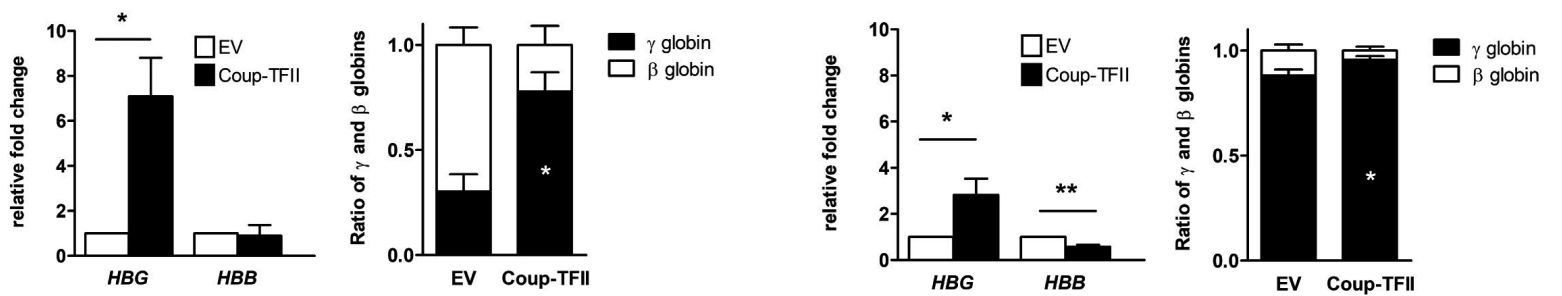

C

human healthy donor
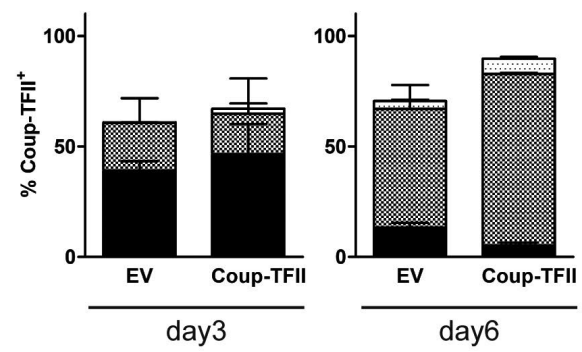

$\mathrm{CD} 71^{+} \mathrm{GpA} \mathrm{A}^{-}$

$\mathrm{CD}^{2} 1^{+} \mathrm{GpA} \mathrm{A}^{+}$

$\mathrm{CD}^{-}{ }^{-} \mathrm{GPA}^{+}$

human thalassemic $\beta^{039}$ patient

Figure 2. Coup-TFIl overexpression increases $\gamma$ - (and $\beta \mathrm{h} 1$ )-globin expression. (A) Quantitative real-time polymerase reaction (RTqPCR) analysis of globin gene expression using cDNA from erythroblasts derived from E13.5 ex-vivo fetal liver erythroid cultures transduced with either empty vector (EV) or with the Coup-TFIl-overexpressing lentiviral particles (Coup-TFII). Left panel: the relative fold-change on the control is shown (error bars: standard error of mean; $* P<0.05$, $* * P<0.01$ ). Expression levels on Gapdh are shown in Online Supplementary Figure S2. Middle panel: the same data are expressed as $\gamma$ to $\beta$ ratio $(\gamma+\beta=1)$. Right panel: RTqPCR analysis for mouse globin genes. In all experiments $>90 \%$ cells were transduced as assessed by flow cytometry (FC) analysis for $\Delta$ NGFR expression (Online Supplementary Figure S2). (B) Coup-TFII overexpression in both healthy and thalassemic $\beta^{\circ} 39$ ex-vivo peripheral blood cultures. The analysis was carried out as in (A). In all experiments, cells were magnetically purified on the basis of $\triangle$ NGFR expression ( $>85 \%$ cells were isolated as assessed by FC analysis on $\triangle N G F R$ expression (Online Supplementary Figure S3). Expression levels on Gapdh are shown in Online Supplementary Figure S3. (C) FC analysis on CD71 and CD235ab (GpA).

This result prompted us to overexpress Coup-TFII in the context of human adult cells, in cultures from peripheral blood from healthy donors and from thalassemic patients carrying the Sardinian $\beta^{0} 39$ mutation, which generates an in-frame stop codon resulting in $\beta$-globin mRNA degradation by nonsense mediated decay. ${ }^{31,32}$ As shown in Figure 2B, Coup-TFII overexpression in normal cells led to a 7-fold increase in $\gamma$-globin RNA expression, with no significant change in $\beta$-globin expression. Similarly, a 3fold activation of $\gamma$-globin was also seen in $\beta^{0} 39$ cells following COUP-TFII overexpression. Of interest, in $\beta^{0} 39$ thalassemic, but not in wild-type cultures, the increase in $\gamma$ expression was accompanied by a decrease in the level of the residual $\beta$ RNA produced from the $\beta^{0} 39$ mutationcarrying alleles, which we tested as a control. Overexpressing Coup-TFII in these cells did not result in statistically significant maturational changes (CD71/GpA profiles in Figure 2C).
Generation of overexpressing and knockout $\beta$-K562 cells

Together, the above data indicate that Coup-TFII is an activator of $\gamma$-globin. To better characterize the role of Coup-TFII at the molecular level, we took advantage of $\beta$ K562 cells, a K562 subclone that, to our knowledge, is the only erythroid cell model system that expresses CoupTFII together with $\varepsilon-, \gamma-$ and $\beta$-globins ${ }^{18}$ (Online Supplementary Figure S4). We used these cells to either knockout by CRISPR/Cas9 (KO cells) or overexpress by lentiviral transduction (OE cells) Coup-TFII (Figure 3A). In $O E$ cells, we observed an increase in embryonic $H B E$ and $H B G$ and a decrease in adult $H B A$ and $H B B$ (Figure $3 B$ ). By contrast, in $\mathrm{KO}$ cells there was a general induction of all globin genes transcription, resulting in increased hemoglobinization (Figure $3 \mathrm{~B}$ and Online Supplementary Figure S6A). In this context, $\beta$-globin expression increased by an average of 45 -fold, whereas the increases in $\alpha$-, $\varepsilon$ - and $\gamma$-globins was about 10 times lower (Figure 3B). This differen- 
A

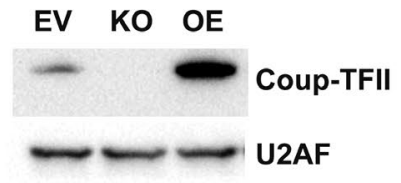

B

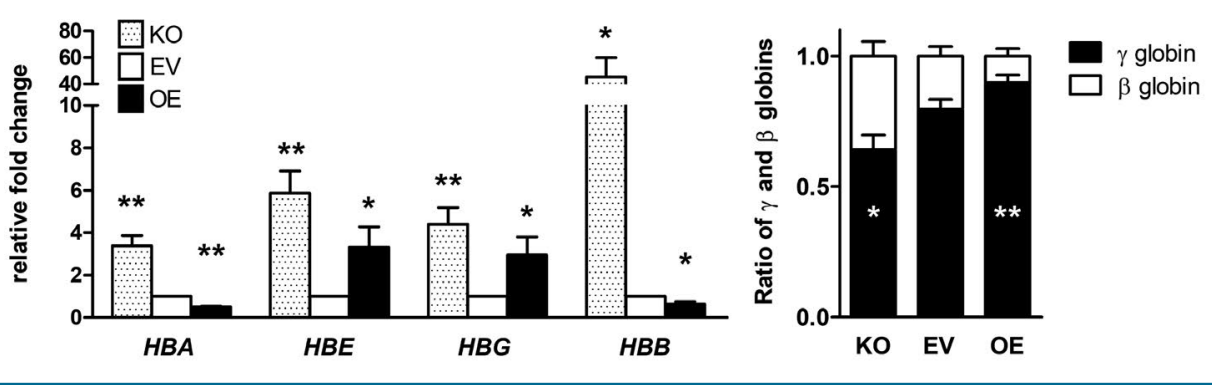

Figure 3. Gain/loss of Coup-TFII expression alters the $\beta$ to $\gamma$ ratio in opposite directions. Coup-TFIl overexpression (OE) was obtained as described in Figure 2; CoupTFII knockout (KO) was achieved using a CRISPR/Cas9 lentiviral vector and the corresponding empty vectors (EV) were used as a control. (A) Western blot confirming the Coup-TFII KO and OE. U2AF: protein loading control. The Coup-TFII KO was also confirmed by quantitative real-time polymerase reaction RTqPCR (not shown). (B) RTqPCR analysis of individual globin genes in a pool of six single clones at day $60(\mathrm{KO})$ and on a pooled population of Coup-TFII OE cells. As a control, a pool of two clones infected with the EV were analyzed. Globin expression levels relative to GAPDH ( $\mathrm{n} \geq 3$, error bars: standard error of mean; *P<0.05) are shown. Right panel: $\gamma-$ to $\beta$-globin ratio $(\gamma+\beta=1)$

A

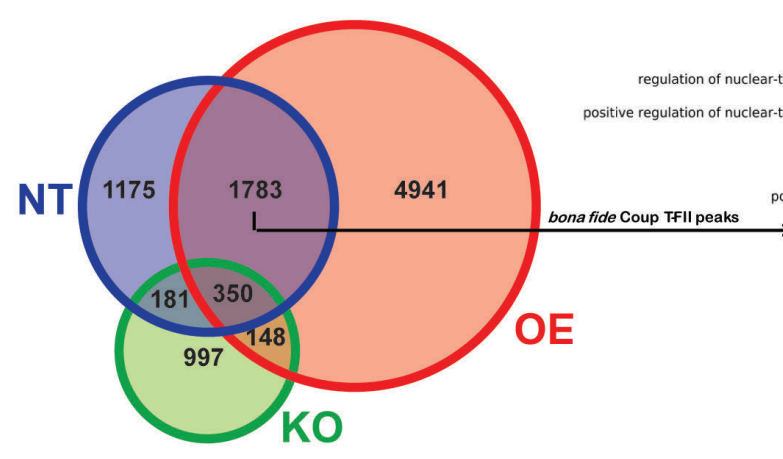

C

\begin{tabular}{ccc}
\hline Motif & E value (-log10) & Centrally enriched \\
\hline & 45,2 & \\
& 5,5 & not centrally enriched \\
\end{tabular}

B

GO Biological Process

- $\log 10$ (Binomial $p$ value)

$\begin{array}{llllllllllll}\text { posttranscriptional regulation of gene expression } & 0 & 1 & 2 & 3 & 4 & 5 & 6 & 7 & 8 & 9 & 9.54\end{array}$ positive requlation of $M R N A$ catabolic process

mmune response-activating signal transductio

bolic process, deadenylation-dependent decar
cellular response

regulation of mRNA stability
response to thyroid hormone stimulus

regulation of RNA stability

regulation of lipid biosynthetic process

histone lysine methylation
protein O-linked glycosylation

protein methylation

cellular response to radiatio

Mouse Phenotype

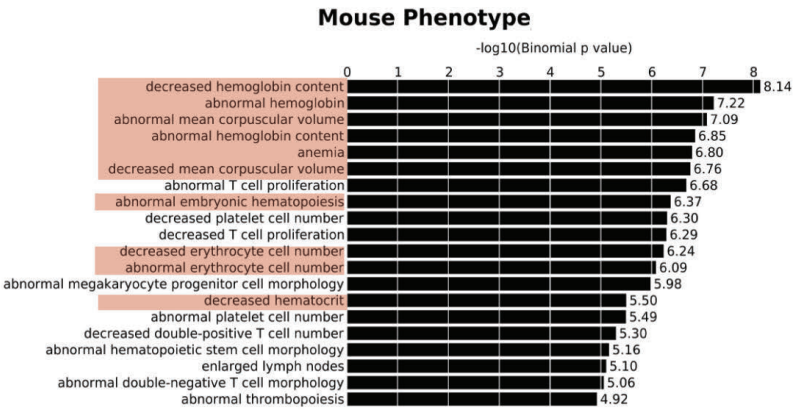

D

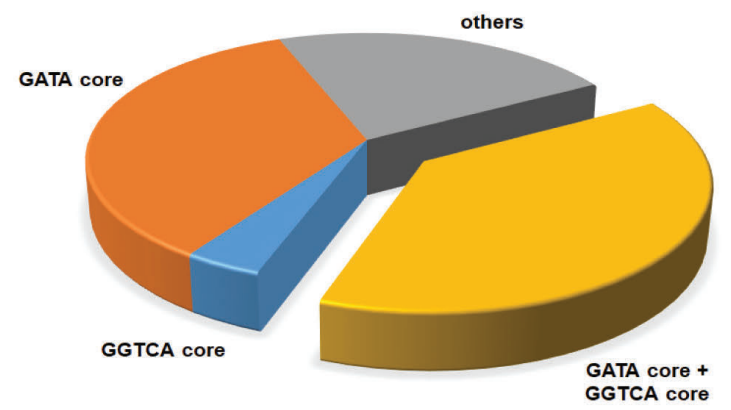

Figure 4. Chromatin immunoprecipitation experiment. (A) Venn diagram illustrating the selection criteria used to identify bona fide Coup-TFII-bound regions. (B) Gene Ontology mouse phenotype association. Erythroid-related phenotypes are shaded in pink. (C) Results of the MEME motif discovery analysis. (D) Pie chart depicting the relative percentages of motifs found by MEME. 
tial increase of $\beta$ versus $\gamma$ levels resulted in an increase in the $\beta /(\gamma+\beta)$ ratio from about $20 \%$ in cells transduced with the empty vector (EV cells) to about $30 \%$ in $\mathrm{KO}$ cells; this is in clear contrast to the decrease in the $\beta /(\gamma+\beta)$ ratio from about $20 \%$ in EV cells to about $8 \%$ in OE cells (Figure $3 \mathrm{~B}$, right panel). This result was confirmed at the protein level (Online Supplementary Figure S6).

\section{Identification of Coup-TFIl in vivo binding sites by} chromatin immunoprecipitation sequencing analysis

To elucidate whether Coup-TFII binds directly to the $\beta$ locus in vivo and to identify other high-confidence relevant Coup-TFII genomic target regions in $\beta-\mathrm{K} 562$ cells, we designed a stringent chromatin immunoprecipitation (ChIP)-sequencing experiment. We selected Coup-TFII peaks common to the $\mathrm{OE}$ and not transduced (NT) cells, from which we subtracted the background peaks present also in KO cells (Figure 4A). Almost $100 \%$ of the remaining 1,783 immunoprecipitated regions mapped to an associated gene and showed a peak distribution profile within -500 and $+500 \mathrm{~kb}$ relative to the transcription start site (Online Supplementary Figure S7). Interestingly, Gene Ontology analysis of mouse phenotypes of genes associated with Coup-TFII peaks clearly pointed to hematologic diseases and in particular to erythroid diseases (shaded in pink in Figure 4B). The MEME algorithm ${ }^{27}$ for enriched motifs discovery identified the canonical (GGTCA) nuclear receptor DNA binding consensus and the GATAfamily (GATAA) consensus as the most represented sequences centered under the Coup-TFII peaks (Figure 4C, D). About $39 \%$ of sequences bound by Coup-TFII, also contain the GATA consensus, suggesting that Coup-TFII does indeed participate in the regulation of the erythroidspecific genetic program. ${ }^{33}$

A

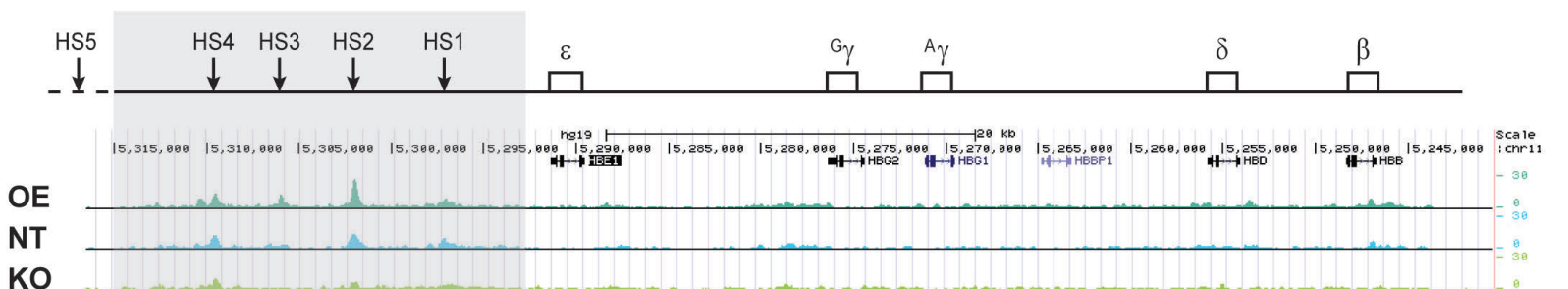

B

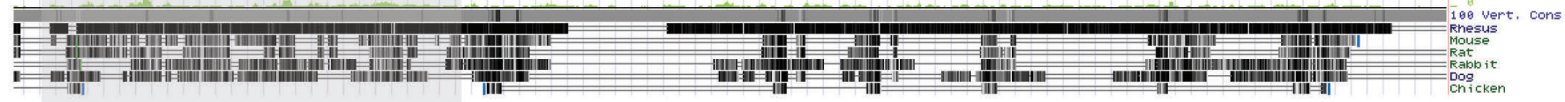

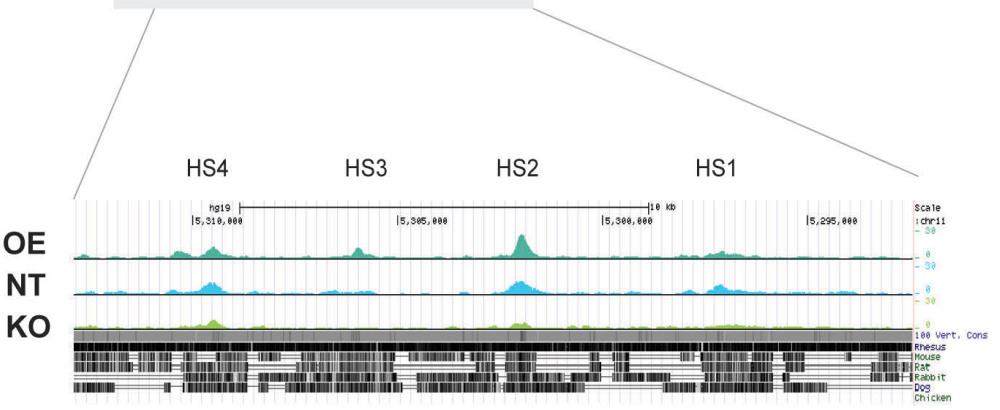

C

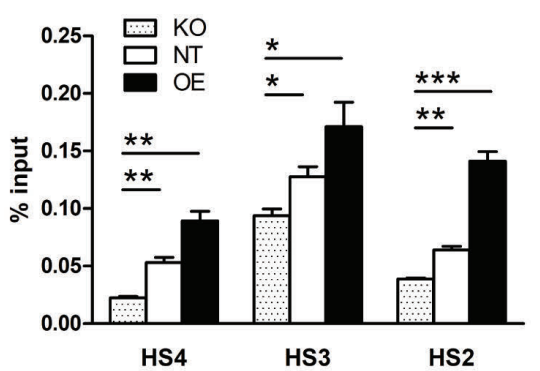

D

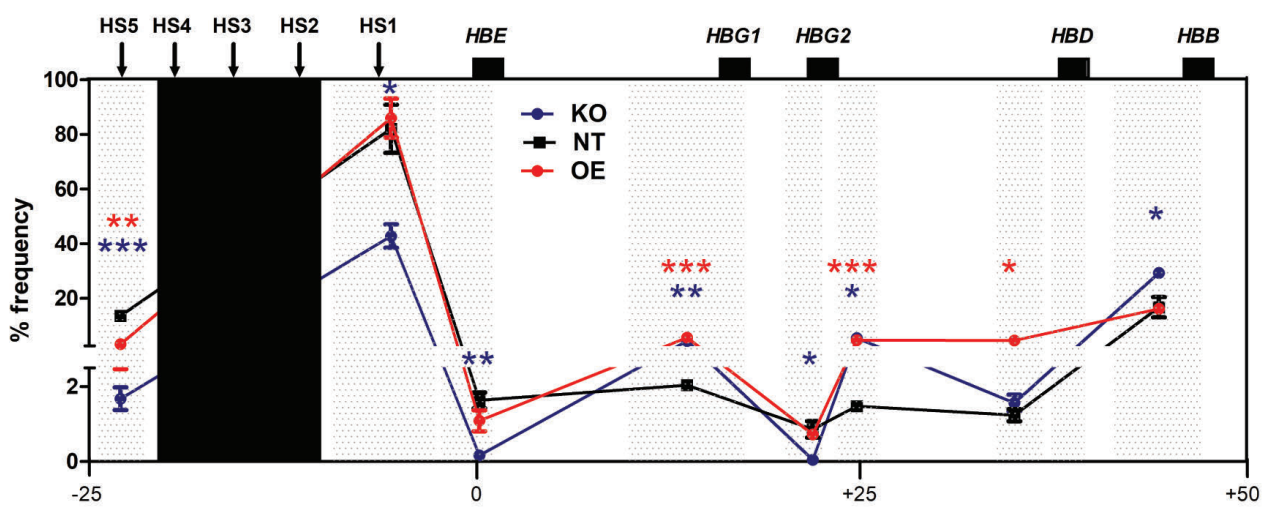

Figure 5. Coup-TFII binds in vivo to the human $\beta$-globin locus. (A) Schematic representation of the human $\beta$-globin locus. (B) Close-up of the HS2, HS3 and HS4 core regions within the locus control region (LCR). (C) Validation of the chromatin immunoprecipitation-sequencing sites within the LCR. (D) Chromosome conformation capture experiment on Coup-TFII-overexpressed (red), not transduced (black) and -knockout (blue) cells. The structure of the locus with the relative positions of globin genes are shown at the top of the Figure. The anchor region is shown in black; the positions and sizes of the analyzed interacting fragments are shaded in light gray. $\left(\mathrm{n}=3\right.$; error bars: standard error of mean; ${ }^{*} P<0.05$; $* * P<0.01$; $\left.* * * P<0.001\right)$. OE: overexpressed; NT: not transduced; KO: knockout. 
Coup-TFII binds in vivo within the human $\beta$-globin locus and contributes to its three-dimensional conformation

The ChIP-sequencing data show that Coup-TFII binds in vivo within the $\beta$-locus, with the strongest peaks (present in both NT and OE cells but absent in KO cells) mapping within the locus control region (LCR), at hypersensitive sites HS2, HS3 and HS4 (Figure 5A, B). Scanning of the core sequence of these elements using JASPAR software ${ }^{34}$ identified several potential Coup-TFII consensus sequences within these elements (Online Supplementary Figure S8), which were confirmed by ChIP assays (Figure $5 \mathrm{~B}, \mathrm{C})$. To determine whether the level of Coup-TFI could influence the chromatin structure of the $\beta$-locus, we performed a $3 \mathrm{C}$ assay on the OE, NT and KO $\beta$-K562 cell lines described above. In this assay, the physical interaction within two nearby loci is measured on the basis of the relative crosslinking efficiency between a predefined anchor region and given downstream positions.

In our experiment, in the absence of Coup-TFII the relative frequency of interactions of the $\gamma$-gene with the LCR is significantly lower $(\approx 4 \%)$ than that of $\beta(\approx 30 \%)$, pointing to an effect on the $\beta$-locus favoring $\beta$-globin expression (in agreement with data in Figure 3B). By contrast, in the presence of Coup-TFII (OE and NT cells), the interaction of $\beta$ with the LCR is around $15 \%$. Of note, in KO cells, the absolute frequencies of interactions within the $\beta$-locus with the LCR increases, possibly reflecting concomitant overlapping differentiation effects.

Of interest, in OE cells, a significant interaction was observed in the A $\gamma-\delta$ region which has been implicated in hereditary persistence of fetal hemoglobin ${ }^{35}$ (Figure 5D). These results, together with ChIP-sequencing data identi- fying Coup-TFI peaks in different regions within the $\beta$ locus, suggest a potential broad role of Coup-TFII in the differential regulation of globin genes and of the $\beta$-locus structure.

\section{Discussion}

In recent years, several repressors of embryonic/fetal globin genes have been identified in murine and human adult erythroid cells. ${ }^{6,36}$ These studies have not directly addressed the regulation of these genes in earlier developmental stages. In particular, there is no evidence so far of stage-specific factors activating human and mouse embryonic/fetal globins prior to the switch to adult globin synthesis. Here we show that Coup-TFII is expressed in cells of yolk sac origin (Figure 1) and that its overexpression selectively activates embyonic/fetal globins in a variety of adult erythroid cells, including $\beta^{0} 39$-thal cells (Figure 2). Coup-TFII overexpression increases $\gamma$-globin relative to $\beta$ globin expression and shows that Coup-TFII is not a repressor of $\gamma$-globin, as previously suggested by in vitro DNA binding studies. ${ }^{37}$

This evidence helps to reconcile the previously unexplained observation that a mutation specifically abolishing Coup-TFII in vitro binding to the $\gamma$ promoter $(-107-108)^{38}$ failed to generate the phenotype of hereditary persistence of fetal hemoglobin in a mouse line transgenic for the human $\beta$-locus. In the absence of Coup-TFII, the $\gamma$ - to $\beta$ globin ratio is decreased, pointing to a possible alternative interaction between $\gamma$ - and $\beta$-globin genes with the LCR. ${ }^{39}$

To answer the question of whether Coup-TFII binds directly to the $\beta$-locus in vivo, we carried out ChIP-

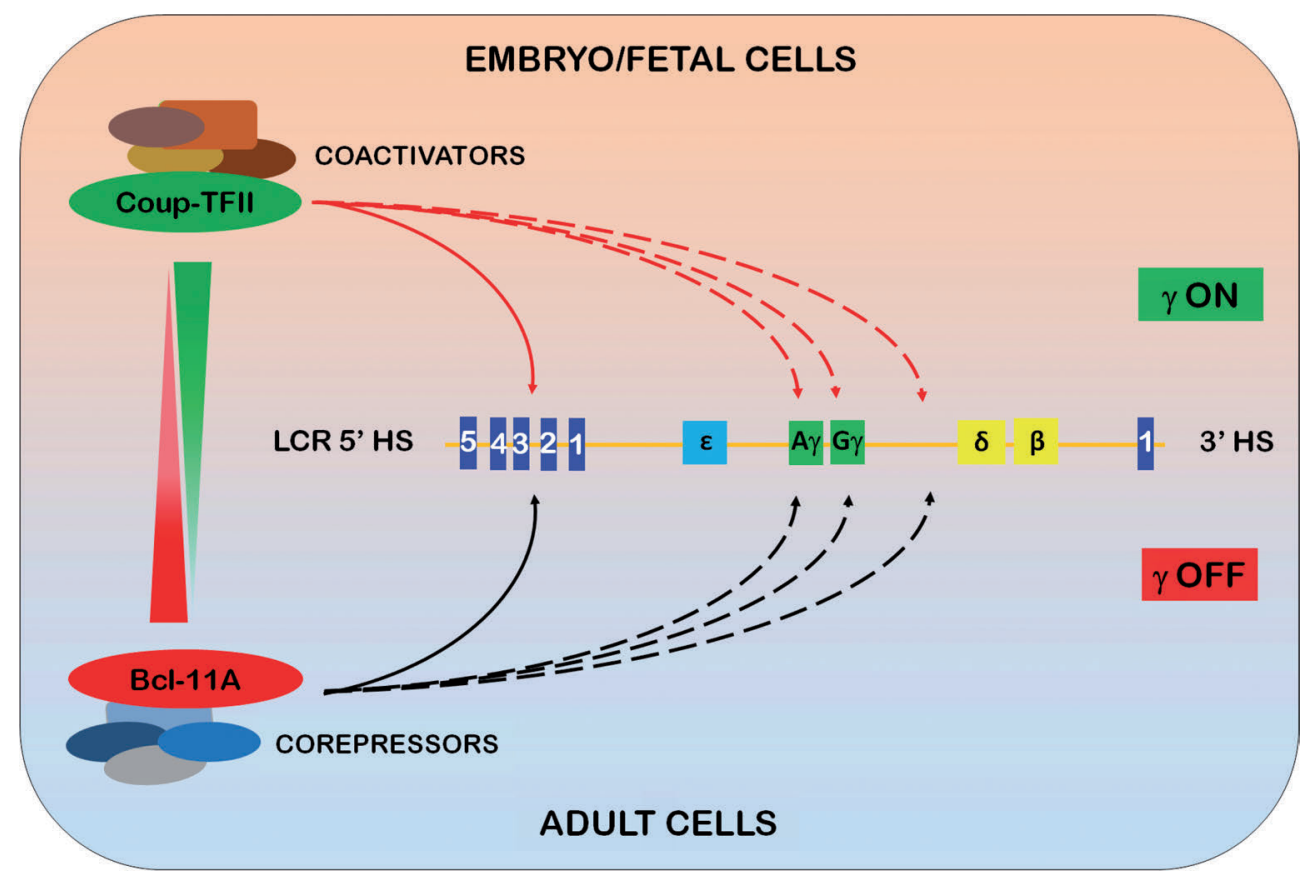

Figure 6. Hypothetical model for the differential $\gamma$-globin activation/silencing in embryonic vs. adult erythroid cells. In embryo/fetal cells, Coup-TFIl is expressed and recognizes the GGTCA consensus sequences within the locus control region (LCR) and possibly at other positions within the $\beta$-locus. The resulting $\beta$-locus architecture favors the expression of $\gamma$-genes. In adult cells, the same positions are occupied by Bcl-11A. The switch between the two factors results in $\gamma$-silencing. The overexpression of Coup-TFII is capable of reverting $\gamma$-silencing in adult cells, suggesting that the erythroid cellular environment is similar in embryo/fetal and adult cell types, possibly because of the presence of a large set of common factors/cofactors. Whether Coup-TFII and Bcl-11A co-exist in some transient cellular stage during development or are distinctly expressed in different cells, remains to be elucidated. Continuous-line arrows point to the strong binding sites within the LCR; dashed-line arrows point to the other possible binding sites within the locus. 
sequencing and $3 \mathrm{C}$ experiments in $\beta-\mathrm{K} 562$ cells, which offer the advantage of co-expression of Coup-TFII together with $\varepsilon$-, $\gamma$ - and $\beta$-globins, making it possible to evaluate the effects of the modulation of Coup-TFII levels. We demonstrated that Coup-TFII does indeed bind the GGTCA binding sites present within the LCR and that its $\mathrm{KO}$ favors LCR- $\beta$-globin interactions (Figure 5 ). The presence of Coup-TFII binding at different positions suggests a possible widespread action of Coup-TFII in the transcriptional regulation of the $\beta$-locus. The functional relevance of these single binding sites will require their precise editing, with the caveat that the presence of overlapping/adjacent binding sites, bound by different transcription factors/cofactors (as in the case of the CCAAT box region of $\gamma$ genes), could complicate the interpretation of the results.

Of interest, the Coup-TFII consensus sequence retrieved by MEME (GGTCA) in ChIP-sequencing almost perfectly matches the one described for Bcl11a-XL ${ }^{40}$ and for TR2/TR4, two known repressors of $\gamma$-globin in adult cells. ${ }^{40,41}$ As for Bcl11a-XL (for which direct binding to the $\gamma$ promoter could only be seen using the very sensitive CUT\&RUN technique ${ }^{40}$ ), we could only detect very weak peaks on the CCAAT box region of the $\gamma$ promoter, although previous in vitro binding and dimethyl sulfate interference experiments clearly mapped the Coup-TFII binding sites to the double CCAAT box $\gamma$-promoter region. ${ }^{14}$ The binding of Coup-TFII to the LCR (and possibly its interaction with the weaker sites on the double CCAAT box region), suggests that it might concur to establish/maintain the architecture of the $\beta$-locus in early developmental stages, when preferential $\gamma$ activation is required, as confirmed by the $3 \mathrm{C}$ experiment (Figure 5). The same regions ( $\gamma$-promoter and LCR) bound by CoupTFII within the LCR, are occupied in adult cells by the $\gamma$ repressors Bcl11a-XL and/or TR2/TR4 that keep $\gamma$-globin expression switched off. Coup-TFII is expressed in an early developmental stage, whereas expression of Bcl11a$\mathrm{XL}$ and TR2/TR4 is confined to adult cells. This would suggest that repression of $\gamma$-globin in the adult stage may involve a switch between factors binding to the CCAAT box within the $\gamma$ promoters and potentially within the LCR, i.e., from Coup-TFII in erythroid cells of yolk sac origin to Bcl11a-XL and TR2/TR4 in adult erythroid cells. The existence of specific developmental activators and repressors could be made necessary by the presence in both embryonic/fetal and adult cells of a common set of activators/coactivators (such as Gata1 and its coactivators) that create a "permissive environment" to which the $\gamma$ promoter is responsive. This hypothesis would also help to explain why Coup-TFII, when overexpressed in adult cells, is indeed capable of activating $\gamma$-globin, overcoming $\gamma$-globin repression (Figure 2).

Whether Coup-TFII and Bcl11a-XL and/or TR2/TR4 coexist in the same cell in a given transitory status in which they directly compete/substitute each other to allow the switch (from $\gamma$ "ON" to $\gamma$ "OFF"), or whether their expression is restricted to non-overlapping spatial and temporal windows of development, remains to be elucidated.

The novel role of Coup-TFII as an activator of fetal globin genes provides new insight into the early activation of embryonic/fetal genes and, possibly, on the mechanisms of hemoglobin switching. In the light of these observations, Coup-TFII could be considered as a target for $\gamma$-globin reactivation. Although COUP-TFII has been extensively studied genetically, present knowledge of upstream regulatory signals and of possible ligands, remains poor. Retinoids, ${ }^{42-44}$ sonic hedgehog, ${ }^{45}$ ETS and their coactivators $^{46}$ activate COUP-TFII expression in different cellular contexts. Moreover, whereas structural data suggest that the Coup-TFII protein is an "orphan" receptor capable of an auto-repressive conformation, there is evidence that Coup-TFII ligands may exist ${ }^{47}$ and, in addition, descriptions of synthetic Coup-TF agonists have been recently published ${ }^{48}$ Collectively, the evidence that Coup-TFII can be transcriptionally induced and functionally activated suggests that it may be an attractive novel target for $\gamma$-globin reactivation in $\beta$-hemoglobinopathies.

\section{Disclosures}

No conflicts of interest to disclose.

\section{Contributions}

SE, CF, GB and MGM performed experiments, and analyzed and discussed data; MFM performed FC analysis; LM performed human cultures; CC performed ChIP-seq experiments; FG performed immunofluorescence analysis; IFM and $S G$ performed experiments. YN provided HUDEP cells; JS contributed with support and discussion of the research; $P M, S O$ and SMLB critically discussed the manuscript; AR led the project and wrote the paper.

\section{Funding}

The authors would like to thank Dr. Andrea Ditadi for help with experimental work, Fondazione Cariplo grant $n$. 2012.0517 for support to AR, PM and JS, the People Programme (Marie Curie Actions) of the European Union's Seventh Framework Programme FP7/2007-2013/ under REA grant agreement n. 289611 (HEM_ID Project) for support to $A R$ and JS and the Knut and Alice Wallenberg foundation for support to CC.

\section{References}

1. Forget BG. Molecular basis of hereditary persistence of fetal hemoglobin. Ann N Y Acad Sci. 1998;850:38-44.

2. Nienhuis AW. Gene transfer into hematopoietic stem cells. Blood Cells. 1994;20(1):141-147; discussion 147-148

3. Thein SL, Craig JE. Genetics of $\mathrm{Hb}$ F/F cell variance in adults and heterocellular hereditary persistence of fetal hemoglobin. Hemoglobin. 1998;22(5-6):401-414.

4. Wahlberg K, Jiang J, Rooks $\mathrm{H}$, et al. The
HBS1L-MYB intergenic interval associated with elevated $\mathrm{HbF}$ levels shows characteristics of a distal regulatory region in erythroid cells. Blood. 2009;114(6):1254-1262.

5. Wilber A, Nienhuis AW, Persons DA Transcriptional regulation of fetal to adult hemoglobin switching: new therapeutic opportunities. Blood. 2011;117(15):39453953.

6. Sankaran VG, Orkin SH. The switch from fetal to adult hemoglobin. Cold Spring Harb Perspect Med. 2013;3(1):a011643.

7. McGrath KE, Frame JM, Fegan KH, et al.
Distinct sources of hematopoietic progenitors emerge before HSCs and provide functional blood cells in the mammalian embryo. Cell Rep. 2015;11(12):1892-1904.

8. McGrath KE, Frame JM, Fromm GJ, et al. A transient definitive erythroid lineage with unique regulation of the $\beta$-globin locus in the mammalian embryo. Blood. 2011;117(17):4600-4608.

9. Pereira FA, Qiu Y, Zhou G, Tsai MJ, Tsai SY The orphan nuclear receptor COUP-TFII is required for angiogenesis and heart development. Genes Dev. 1999;13(8):1037-1049. 
C. Fugazza et al.

10. Lin FJ, Gin J, Tang K, Tsai SY, Tai MJ. Coup d'etat: an orphan takes control. Endocr Rev. 2011;32(3):404-421.

11. Filipe A, Li O, Deveaux S, et al. Regulation of embryonic/fetal globin genes by nuclear hormone receptors: a novel perspective on hemoglobin switching. EMBO J. 1999;18(3): 687-697.

12. Cur S, Tanabe $O$, Sierant $M$, et al. Compound loss of function of nuclear receptors $\operatorname{Tr} 2$ and $\operatorname{Tr} 4$ leads to induction of murine embryonic $\beta$-type globin genes. Blood. 2015;125(9):1477-1487.

13. Liberati C, Ranchi A, Lievens P, Ottolenghi S, Mantovani R. NF-Y organizes the gamma-globin CCAAT boxes region. J Biol Chem. 1998;273(27):16880-16889.

14. Ronchi AE, Bottardi S, Mazzucchelli C, Ottolenghi S, Santoro C. Differential binding of the NFE3 and CP1/NFY transcription factors to the human gamma- and epsilonglobin CCAAT boxes. J Biol Chem. 1995;270(37):21934-21941.

15. Liberati C, Gera MR, Secco P, et al. Cooperation and competition between the binding of COUP-TFII and NF-Y on human epsilon- and gamma-globin gene promoters. J Biol Chem. 2001;276(45):41700-41709.

16. Gui S, Kolodziej KE, Obara N, et al. Nuclear receptors TR2 and TR4 recruit multiple epgenetic transcriptional corepressors that associate specifically with the embryonic $\beta$ type globin promoters in differentiated adult erythroid cells. Mol Cell Biol. 2011;31(16): 3298-3311.

17. Zhu X, Wang Y, Pi W, Lu H, Wickrema A, Juan D. NF-Y recruits both transcription activator and repressor to modulate tissueand developmental stage-specific expression of human $\gamma$-globin gene. CLoS One. 2012;7(10):e47175.

18. Durlak M, Fugazza C, Elangovan S, et al. A novel high-content immunofluorescence assay as a tool to identify at the single cell level $\gamma$-globin inducing compounds. CLoS One. 2015;10(10):e0141083

19. ven Lindern M, Diner EM, Dolznig H, et al. Leukemic transformation of normal murine erythroid progenitors: $\mathrm{v}$ - and c-ErbB act through signaling pathways activated by the EpoR and c-Kit in stress erythropoiesis. Oncogene. 2001;20(28):3651-3664.

20. Migliaccio G, Di Pietro R, Di Giacomo V, et al. In vitro mass production of human rytheroid cells from the blood of normal donors and thalassemic patients. Blood Cells Mol Dis. 2002;28(2):169-180.

21. Schreiber E, Matthias P, Muller MM, Schaffner W. Rapid detection of octamer binding proteins with 'mini-extracts', pres- pared from a small number of cells. Nucleic Acids Res. 1989;17(15):6419.

22. Afgan E, Baker D, van den Beek M, et al. The Galaxy platform for accessible, reproducible and collaborative biomedical analysex: 2016 update. Nucleic Acids Res. 2016;44(W1):W3-W10.

23. Li H, Durbin R. Fast and accurate short read alignment with Burrows-Wheeler transform. Bioinformatics. 2009;25(14):1754-1760.

24. Heinz S, Brenner C, Stan N, et al. Simple combinations of lineage-determining transcription factors prime cis-regulatory lemints required for macrophage and $\mathrm{B}$ cell identities. Mol Cell. 2010;38(4):576-589.

25. Ramirez F, Dundar F, Diehl S, Gruning BA, Manke T. deepTools: a flexible platform for exploring deep-sequencing data. Nucleic Acids Res. 2014;42(Web Server issue):W187191.

26. Quintan AR, Hall IM. BEDTools: a flexible suite of utilities for comparing genomic facures. Bioinformatics. 2010;26(6):841-842.

27. Machanick P, Bailey TL. MEME-ChIP: motif analysis of large DNA datasets. Bioinformatics. 2011;27(12):1696-1697.

28. Palstra RJ, Tolhuis B, Splinter E, Nijmeijer R, Grosveld F, de Lat W. The beta-globin nuclear compartment in development and erythroid differentiation. Nat Genet. 2003;35(2):190-194.

29. Xu J, Sankaran VG, Ni M, et al. Transcriptional silencing of $\gamma$-globin by BCL11A involves long-range interactions and cooperation with SOX6. Genes Nev. 2010;24(8):783-798.

30. Patrinos GP, de Krom M, de Boer E, et al. Multiple interactions between regulatory regions are required to stabilize an active chromatin hub. Genes Nev. 2004;18(12): 1495-1509.

31. Gao A, Galanello R, Furbetta M, et al. Thalassaemia types and their incidence in Sardinia. J Med Genet. 1978;15(6):443-447.

32. Baserga SJ, Benz EJ, Jr. Beta-globin nonsense mutation: deficient accumulation of mRA occurs despite normal cytoplasmic stability. Proc Natl Acad Sci U S A. 1992;89(7):29352939.

33. Eros E, Balint BL. COUP-TFII is a modulator of cell-type-specific genetic programs based on genomic localization maps. J Biotechnol. 2019;301:11-17.

34. Mathelier A, Fornes O, Arenillas DJ, et al. JASPAR 2016: a major expansion and update of the open-access database of transcription factor binding profiles. Nucleic Acids Res. 2016;44(D1):D110-115.

35. Highs DR, Engel JD, Stamatoyannopoulos G. Thalassaemia. Lancet. 2012;379(9813):
373-383.

36. Suzuki M, Yamamoto M, Engel JD. Fetal globin gene repressors as drug targets for molecular therapies to treat the $\beta$-grobinopathies. Mol Cell Biol. 2014;34(19): 3560-3569.

37. Ottolenghi S, Nicolis S, Bertini C, Ranchi A, Cotta S, Giglioni B. Regulation of gammaglobin expression in hereditary persistence of fetal hemoglobin. Ann N Y Acid Sci. 1990;612:191-195.

38. Ronchi A, Berry M, Raguz S, et al. Role of the duplicated CCAAT box region in gamma-globin gene regulation and hereditary persistence of fetal haemoglobin. EMBO J. 1996;15(1):143-149.

39. Wijgerde M, Grosveld F, Fraser P. Transcription complex stability and chromatin dynamics in vive. Nature. 1995;377 (6546):209-213.

40. Lu N, Hargreaves VV, Zhu Q, et al. Direct promoter repression by BCL11A controls the fetal to adult hemoglobin switch. Cell. 2018;173(2):430-442.

41. Tanabe O, McPhee D, Kobayashi S, et al. Embryonic and fetal beta-globin gene repression by the orphan nuclear receptors, TR2 and TR4. EMBO J. 2007;26(9):2295-2306.

42. Jonk LJ, de Jorge ME, Vervaart JM, Wissink $\mathrm{S}$, Kruijer W. Isolation and developmental expression of retinoic-acid-induced genes. Div Biol. 1994;161(2):604-614.

43. Diu Y, Krishna V, Pereira FA, Tsai SY, Tsai MJ. Chicken ovalbumin upstream promoter-transcription factors and their regulation. J Steroid Biochem Mol Biol. 1996;56(1-6 Spec No):81-85.

44. Soosaar A, Newman K, Nornes HO, Newman T. Cell type specific regulation of COUP-TF II promoter activity. FEBS Lett. 1996;391(1-2):95-100.

45. Krishnan V, Elberg G, Tai MJ, Tsai SY. Identification of a novel sonic hedgehog response element in the chicken ovalbumin upstream promoter-transcription factor II promoter. Mol Endocrinol. 1997;11(10): 1458-1466.

46. Petit FG, Salas R, Tai MJ, Thai SY. The regulation of COUP-TFII gene expression by Ets-1 is enhanced by the steroid receptor coactivators. Mech Ageing Nev. 2004;125(1011):719-732.

47. Kruse SW, Suino-Powell K, Zhou XE, et al. Identification of COUP-TFII orphan nuclear receptor as a retinoic acid-activated receptor. CLoS Biol. 2008;6(9):e227.

48. Yoon K, Chen CC, Orr AA, Barreto PN, Tamamis P, Safe S. Activation of COUP-TFI by a novel diindolylmethane derivative. Cells. 2019;8(3):220.

482

haematological | 2021; 106(2) 